\title{
Giant fundic gland polyp mimicking a gastric malignancy
}

A 67-year-old man presented with nonspecific abdominal discomfort. Gastroscopy showed a polypoid mass carpeting the lesser curvature and large parts of the anterior wall of the gastric body (Figure 1a). On endosonography the tumor was found to be partially cystic, with irregular thickening of the first three layers (Figure 1b). Parts of the lesion were resected endoscopically. Histological examination revealed the typical features of a benign fundic gland polyp, with cystically dilated glands lined by flattened parietal cells, chief cells, and occasional mucinous cells; there was no nuclear atypia and no mitotic figures (Figure 1c, d). A single low-grade tubular adenoma was found in the patient's sigmoid colon. His family history was unremarkable.

Fundic gland polyps account for approximately $50 \%$ of all gastric polyps, and they usually range in size from $1 \mathrm{~mm}$ to $5 \mathrm{~mm}$ [1]. Their pathogenesis is poorly understood. Most are sporadic, though they are found in up to $80 \%-93 \%$ of patients with familial adenomatous polyposis. In such patients, low-grade intraepithelial neoplasia and/or foveolar microadenomas may be present in $44 \%-54 \%$ of cases [2]. In addition, fundic gland polyps have been related to proton-pump inhibitor therapy [3], although the data on this are conflicting [4].

This case report is the first to describe a giant gastric fundic gland polyp with endosonographic features that mimicked a malignant lesion. The benign nature of this polyp was confirmed by histological examination, and no foci of neoplastic and/or microadenomatous change were observed. The pathogenesis of this particular lesion remains unclear: the patient was not taking proton-pump inhibitors and there was no evidence of familial adenomatous polyposis.

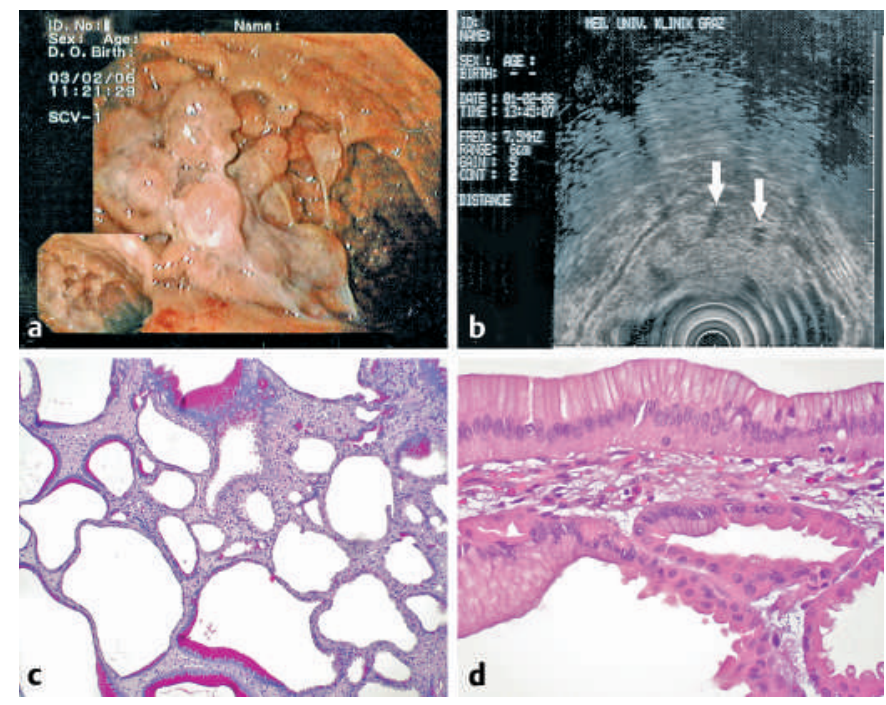

Figure 1 Giant gastric fundic gland polyp. The endoscopic view showed a large, partly cystic, polypoid mass covering large parts of the gastric body (a). Endosonography showed a tumor with cyst formation (arrows) and thickening of the first three layers, but an intact muscularis propria (b). Histological examination revealed benign, cystically dilated glands after staining with periodic acid-Schiff stain (c). On hematoxylin and eosin staining, the glands can be seen to be lined by flattened parietal cells, chief cells, and mucinous cells, with no nuclear atypia (d).

\section{A. Winkler ${ }^{1}$, T.A. Hinterleitner ${ }^{2}$, C. Langner ${ }^{1}$}

${ }^{1}$ Institute of Pathology, Medical University of Graz, Graz, Austria

2 Department of Internal Medicine, Medical University of Graz, Graz, Austria.

\section{References}

${ }^{1}$ Weston BR, Helper DJ, Rex DK. Positive predictive value of endoscopic features deemed typical of gastric fundic gland polyps. J Clin Gastroenterol 2003; 36: 399-402

2 Stolte M, Vieth M, Ebert MP. High-grade dysplasia in sporadic fundic gland polyps: clinically relevant or not? Eur J Gastroenterol Hepatol 2003; 15: 1153-1156

3 Burt RW. Gastric fundic gland polyps. Gastroenterology 2003; 125: $1462-1469$

${ }^{4}$ Vieth M, Stolte M. Fundic gland polyps are not induced by proton pump inhibitor therapy. Am J Clin Pathol 2001; 116: 716-720
Corresponding author

\section{Langner, M.D.}

Institute of Pathology

Medical University Graz

Auenbruggerplatz 25

A-8036 Graz

Austria

Fax: $\quad+43-316-384329$

Email: cord.langner@meduni-graz.at 